\title{
Anatomical and functional responses in eyes with diabetic macular edema treated with "1 + PRN" ranibizumab: one-year outcomes in population of mainland China
}

Kunbei Lai ${ }^{\dagger}$, Chuangxin Huang ${ }^{\dagger}$, Longhui Li, Yajun Gong, Fabao Xu, Xiaojing Zhong, Lin Lu and Chenjin Jin ${ }^{*}$ (D)

\begin{abstract}
Background: To evaluate the anatomical and functional responses in eyes with diabetic macular edema (DME) treated with ranibizumab under "1 + pro re nata (PRN)" regimen.

Methods: This prospective interventional case series included 69 eyes of 69 patients with DME treated with intravitreal injections of $0.5 \mathrm{mg}$ ranibizumab followed by repeated injections as needed. Best-corrected visual acuity (BCVA), central foveal thickness (CFT), subfoveal choroidal thickness (SFCT), and predictive factors for final visual outcomes were assessed.

Results: Logarithm of minimal angle of resolution (logMAR) BCVA improved from $0.64 \pm 0.23$ at baseline to $0.56 \pm$ $0.27,0.53 \pm 0.26,0.47 \pm 0.25,0.44 \pm 0.32,0.47 \pm 0.26$ and $0.46 \pm 0.26$ at time-point of months $1,2,3,6,9$, and 12 , respectively $(P<0.05$ for any follow-up time-point except month 1). CFT decreased from $478.23 \pm 172.31 \mu \mathrm{m}$ at baseline to $349.74 \pm 82.21 \mu \mathrm{m}, 313.52 \pm 69.62 \mu \mathrm{m}, 292.59 \pm 61.07 \mu \mathrm{m}, 284.67 \pm 69.85 \mu \mathrm{m}, 268.33 \pm 43.03 \mu \mathrm{m}$, and $270.39 \pm 49.27 \mu \mathrm{m}$ at above time-points, respectively $(P<0.05)$. The number of injections was 6.83 times over 12 months' follow-up under " $1+P R N$ " regimen. Multivariate analysis showed that the factors including age, BCVA at baseline, disruption of ellipsoid zone, posterior vitreous detachment (PVD), and vitreomacular traction (VMT) were correlated with the final BCVA.
\end{abstract}

Conclusions: Intravitreal injections of ranibizumab under " $1+$ PRN" regimen is a not only effective but also safe way to improve visual acuity of DME patients. And older age, lower baseline BCVA, VMT, and disruption of ellipsoid zone are predictors for final poor BCVA while PVD is a positive predictive factor for good final BCVA.

Trial registration: The trial was registered retrospectively in ClinicalTrials.gov on 2 June 2019 (NCT03973138).

Keywords: Diabetic macular edema, Ranibizumab, Central foveal thickness, Predictive factor, Pro re nata, Subfoveal choroidal thickness

\footnotetext{
* Correspondence: jinchj@mail.sysu.edu.cn

${ }^{\dagger}$ Kunbei Lai and Chuangxin Huang contributed equally to this work.

State Key Laboratory of Ophthalmology, Zhongshan Ophthalmic Center, Sun

Yat-sen University, 54 South Xianlie Road, Guangzhou 510060, China
}

(C) The Author(s). 2020 Open Access This article is licensed under a Creative Commons Attribution 4.0 International License, which permits use, sharing, adaptation, distribution and reproduction in any medium or format, as long as you give appropriate credit to the original author(s) and the source, provide a link to the Creative Commons licence, and indicate if changes were made. The images or other third party material in this article are included in the article's Creative Commons licence, unless indicated otherwise in a credit line to the material. If material is not included in the article's Creative Commons licence and your intended use is not permitted by statutory regulation or exceeds the permitted use, you will need to obtain permission directly from the copyright holder. To view a copy of this licence, visit http://creativecommons.org/licenses/by/4.0/. The Creative Commons Public Domain Dedication waiver (http://creativecommons.org/publicdomain/zero/1.0/) applies to the data made available in this article, unless otherwise stated in a credit line to the data. 


\section{Background}

Diabetic macular edema (DME) is an important cause of visual impairment in patients with diabetes mellitus (DM), which affects greatly the quality of individual's life [1-3]. Since the prevalence of diabetes worldwide is increasing, DME has become a global health issue $[4,5]$. Laser photocoagulation has once been the standard treatment protocol for DME during the past three decades [6]. Nowadays, treatment of DME shifts to antivascular endothelial growth factor (anti-VEGF) therapy [7]. Recent randomized multicenter clinical trials have showed the benefits of anti-VEGF therapy on reducing DME and improving patient's vision [8-11].

Up to now, most of the studies recommend three loading doses of anti-VEGF injections followed by an as needed/pro re nata (PRN) regimen. However, three loading injections would be a great economic and psychological burden for patients with DME, especially for those who in developing countries. Meanwhile, a proportion of patients actually do not need three loading injections and yet maintain good vision over long period. Therefore, "1 + PRN" regimen, namely one anti-VEGF injection at the very first month, followed by an as needed retreatment protocol might be a treatment option for DME. However, this concept of “ $1+\mathrm{PRN}$ ” regimen has not been well studied. Herein, we reported the functional as well as anatomical responses in eyes with DME treated with ranibizumab under the " $1+$ PRN" regimen.

\section{Methods}

\section{Patients}

This study was a prospective, single-center, and interventional study. Prior approval was obtained from the IRB (Institutional Review Board) of Zhongshan Ophthalmic Center of Sun Yat-sen University and all the performance was done in accordance with the tenets of the Declaration of Helsinki. A total of seventy-four patients treated at our hospital (Zhongshan Ophthalmic Center) met the inclusion criteria, however, five patients declined to participate the study for personal reasons, and finally sixty-nine eyes of sixty-nine patients with DME treated at our hospital from January 2015 through June 2019 were enrolled in this study (if both eyes of one patient met the inclusion criteria, the eye with worse VA would enroll in this study). Written informed consents were obtained from all the enrolled patients. The inclusion criteria were: 1) patients aged than 18 years with centerinvolved DME due to with type 1 or $2 \mathrm{DM}$ who had a best-corrected visual acuity (BCVA) between 20/32 and $20 / 200$, and CFT $\geq 300 \mu \mathrm{m}$; 2) DME confirmed by fundus fluorescein angiography (FFA) as well as optical coherence tomography (OCT); 3) decreased vision caused by DME but not any other cause. The exclusion criteria were as follows: 1) previous treatment for DME such as anti-VEGF or laser photocoagulation; 2) patients who required immediate surgery, for example, serious proliferative diabetic retinopathy; 3) other ocular diseases, such as glaucoma, retinal detachment or uveitis; 4) unstable systemic conditions. Our study was registered on https://clinicaltrials.gov (NCT03973138).

\section{Treatment and follow-up}

DME patients were treated by intravitreal injection of ranibizumab (IVR, $0.5 \mathrm{mg}$; Genentech, USA) at the very first month, followed by a protocol of as needed reinjections until stable vision was achieved over 2 consecutive visits or a BCVA of 20/20 was observed. BCVA, slitlamp examination, intraocular pressure (IOP) with tonometry, funduscopy, as well as enhanced depth imaging (EDI)-OCT were performed at baseline and monthly routinely. FFA was performed before treatment and when the surgeon considered it was necessary. The primary outcome was the mean change of BCVA at month 12. The secondary outcomes measured the central foveal thickness (CFT), numbers of injections, predictive factors for final BCVA, as well as systemic/ocular adverse events.

\section{Assessment}

Decimal BCVA were transferred to the logarithm of minimal angle of resolution (logMAR) value for analysis. The CFT was determined by the averaging the foveal thickness of the retinal pigment epithelium (RPE) line to inner retinal surface of vertical scan and horizontal scan [12]. Subfoveal choroidal thickness (SFCT) was determined by the vertical distance from the RPE line to the inner surface of choroidal-scleral junction, as previously described [13]. Patients' characteristics, including the age of onset, gender, duration of DM, duration of DME, BCVA at baseline, CFT, SFCT, continuity of ellipsoid zone (EZ) and external limiting membrane (ELM), as well as other anatomic characteristics of OCT (such as subretinal fluid, intraretinal cysts, posterior vitreous detachment [PVD], and vitreomacular traction [VMT]) at baseline were documented and were analyzed whether they were the predictive factors for one-year visual outcome.

\section{Statistical analysis}

All data was expressed as means \pm SD. SPSS 16.0 software (SPSS Corporation) was used for statistical analyses in our study. The $t$ test and $\chi^{2}$ test were used for continuous variables and categorical variables, respectively. Repeated measure ANOVA was used for the analysis of logMAR VA and CFT. Multivariate analysis was used for the analysis of predictive factors. A $P<0.05$ (two-tailed) was considered statistically significant for all analysis. 


\section{Results}

\section{Patient characteristics}

Seventy-four patients who met inclusion criteria, five patients declined to participate the study for personal reasons, and finally sixty-nine eyes of sixty-nine patients enrolled in our study (Fig. 1). Table 1 showed the baseline characteristics of all enrolled cases. There was no statistical difference for the BCVA at baseline between the intact EZ sub-group and the disrupted EZ sub-group $(0.61 \pm 0.24$ vs $0.66 \pm 0.22) \quad(P>0.05)$. Similar results were found for the BCVA at baseline between the intact ELM group and the disrupted ELM group $(0.60 \pm 0.24 \mathrm{vs}$ $0.68 \pm 0.22)(P>0.05)$. There was no statistical difference for CFT at baseline between subgroups. Thirty-five eyes $(50.72 \%)$ were mild to moderate non-proliferative diabetic retinopathy (NPDR), twenty-one (30.43\%) eyes were severe NPDR, and thirteen eyes (18.84\%) were proliferative DR.

\section{Visual outcome after IVR}

The mean numbers of IVR were 6.83 times during the 12-month follow-up visit. The logMAR VA improved from $0.64 \pm 0.23$ at the baseline to $0.56 \pm 0.27,0.53 \pm$ $0.26,0.47 \pm 0.25,0.44 \pm 0.32,0.47 \pm 0.26$ and $0.46 \pm 0.26$ at the time-points of months $1,2,3,6,9$, and 12, respectively. Significant differences were found for the logMAR VA at any follow-up compared with that of baseline except the time-point of month $1(P<0.05$ for any follow-up time-point except month 1) (Fig. 2a). Interestingly, for sub-group analysis, the mean BCVA between the intact EZ group and disrupted EZ group was compared, although there was no statically difference at the baseline between the intact EZ group and

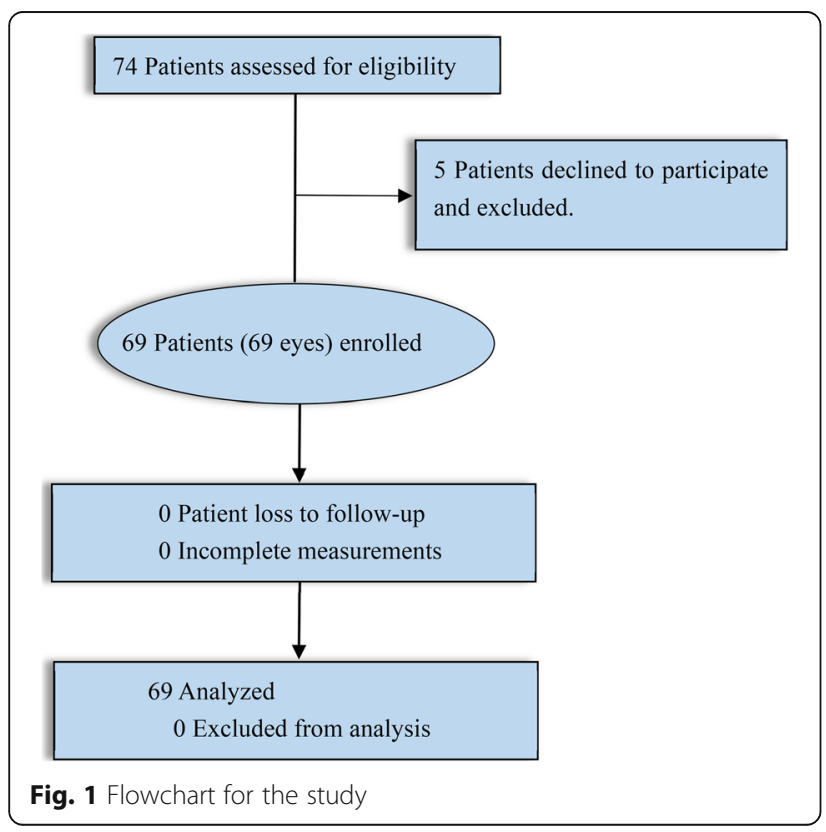

Table 1 The baseline characteristics of the study patients with diabetic macular edema

\begin{tabular}{ll}
\hline Baseline characteristics & $n=69$ \\
\hline Mean age of onset, mean \pm SD & $\begin{array}{l}55.75 \pm 12.17 \\
\text { (range, 22-78) }\end{array}$ \\
Gender & \\
Male, no. (\%) & $41(59.42)$ \\
Female, no. (\%) & $28(40.58)$ \\
BCVA (logMAR units), total, mean \pm SD & $0.64 \pm 0.24$ \\
Sub-group of intact EZ & $0.61 \pm 0.24$ \\
Sub-group of disrupted EZ & $0.66 \pm 0.22$ \\
Sub-group of intact ELM & $0.60 \pm 0.24$ \\
Sub-group of disrupted ELM & $0.68 \pm 0.22$ \\
Central foveal thickness ( $\mu$ m), total, mean \pm SD & $478.23 \pm 172.32$ \\
Sub-group of intact EZ & $476.56 \pm 156.02$ \\
Sub-group of disrupted EZ & $480.40 \pm 194.21$ \\
Sub-group of intact ELM & $481.16 \pm 181.04$ \\
Sub-group of disrupted ELM & $473.38 \pm 160.20$ \\
Subfoveal choroidal thickness( $\mu$ m), mean $\pm S D$ & $229.55 \pm 65.07$ \\
Percentage of intraretinal fluid cyst, no. (\%) & $45(65.22)$ \\
Percentage of subretinal fluid, no. (\%) & $44(63.77)$ \\
Percentage of disrupted EZ, no. (\%) & $30(43.48)$ \\
Percentage of disrupted ELM, no. (\%) & $26(37.68)$ \\
\hline BCVA best-cor
\end{tabular}

$B C V A$ best-corrected visual acuity, logMAR logarithm of the minimal angle of resolution, EZ epiretinal membrane, ELM external limiting membrane

the disrupted EZ group, we found that the final BCVA was better in the intact EZ group than that of disrupted EZ group $(0.61 \pm 0.24$ vs $0.66 \pm 0.22$ at the baseline and $0.39 \pm 0.24$ vs $0.56 \pm 0.26$ at the final visits $)(P<0.01)$. At month 3,7 eyes $(10.14 \%)$ underwent only 1 intravitreal injection, 18 eyes (26.09\%) had 2 injections, and the left 44 eyes $(63.77 \%)$ had 3 injections.

\section{Central foveal thickness after intravitreal injections of ranibizumab}

Mean \pm SD central foveal thickness was $478.23 \pm 172.31 \mu \mathrm{m}$ at baseline, and it decreased to $349.74 \pm 82.21 \mu \mathrm{m}, 313.52 \pm$ $69.62 \mu \mathrm{m}, 292.59 \pm 61.07 \mu \mathrm{m}, 284.67 \pm 69.85 \mu \mathrm{m}, 268.33 \pm$ $43.03 \mu \mathrm{m}$, and $270.39 \pm 49.27 \mu \mathrm{m}$ at above follow-up timepoints, respectively $(P<0.05)$. But there was no difference neither between the intact EZ sub-group and the disrupted EZ sub-group nor between the intact ELM sub-group and the disrupted ELM sub-group at any time-point $(P>0.05)$ (Fig. 2b). Representative images are shown in Fig. 3.

\section{Subfoveal choroidal thickness after IVR}

SFCT at baseline and one-year after IVR were compared in this study, although there was statistical difference between the SFCT at baseline and SFCT at month 12 after IVR under “1 + PRN" regimen $(229.55 \pm 65.07 \mu \mathrm{m}$ vs 

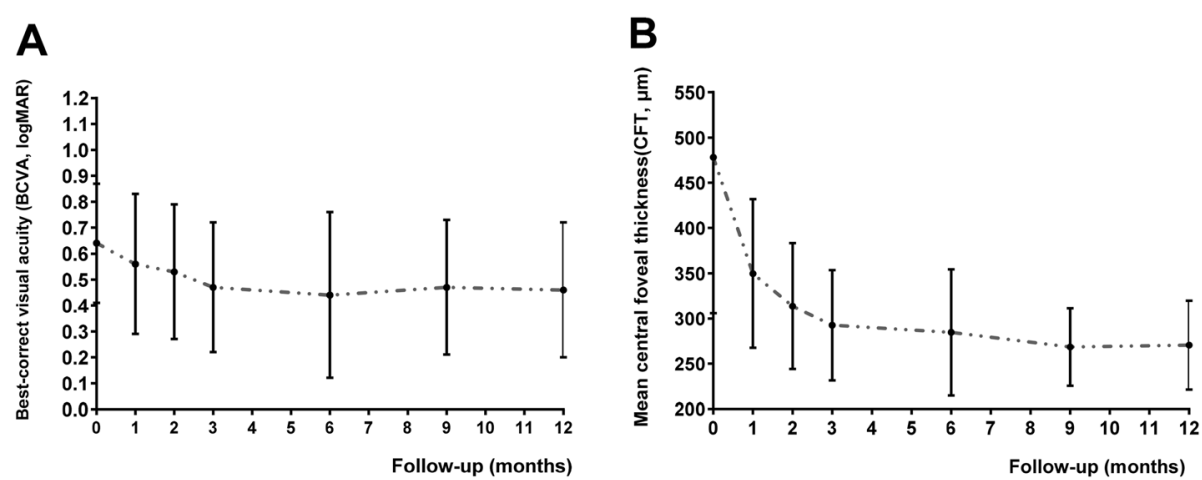

Fig. 2 The changes of the mean logarithm of the minimum angle of resolution (logMAR) visual acuity (VA) and central foveal thickness (CFT) during the 12-month follow-up treated with intravitreal injections of ranibizumab (IVR) under "1 + PRN" regimen. a showed that logMAR VA decreased with follow-up time-point, there were significant differences for the logMAR VA at each time-point compared with the baseline except month 1 ( $P>0.05$ for month 1, $P<0.05$ for other follow-up time-points), (b) showed that " $1+$ PRN" IVR treatment significantly reduced the CFT ( $P<0.05$ for all time-points compared with the baseline)

$209.91 \pm 63.74 \mu \mathrm{m}, P<0.05)$, multivariate linear regression analysis demonstrated that SFCT at baseline was not a predictive factor for BCVA at one-year follow-up $(P>0.05)$.

\section{Predictive factors for one-year visual prognosis}

Multivariate linear regression analysis demonstrated that the predictors for final VA were the age $(P=0.013)$, presence of VMT $(P=0.005)$, BCVA at baseline $(P=$ $0.001)$, PVD development $(P=0.004)$, and EZ disruption $(P=0.01)$ (Table 2). Older age, poor baseline BCVA, presence of VMT, as well as EZ disruption were more at risk of poor final VA than eyes without these findings, while development of PVD was associated with good final VA.

\section{Ocular/systemic complications}

No systemic complication was found in the study. Although there were three patients who experienced transient IOP elevation which became normal on the second day, other ocular complication was not detected in any of the patients.

\section{Discussion}

In this present study, our data showed that intravitreal injections of ranibizumab under " $1+\mathrm{PRN}$ " regimen led to significant improvements in BCVA and reduction of the CFT over 12 months. Besides, our data revealed that older age, poor baseline BCVA, presence of VMT, as well as EZ disruption were more at risk of poor final VA than eyes without these findings, while development of PVD was associated with good final VA at month 12.

VEGF is an important mediator which responses for the abnormal vascular permeability in DME [14, 15]. Ranibizumab, a recombinant humanized monoclonal antibody for VEGF-A [16, 17], was approved by FDA for indication of DME in 2012. Many clinical trials, including READ-2 study [18, 19], the RESOLVE study [9, 20], the Diabetic Retinopathy Clinical Research Network

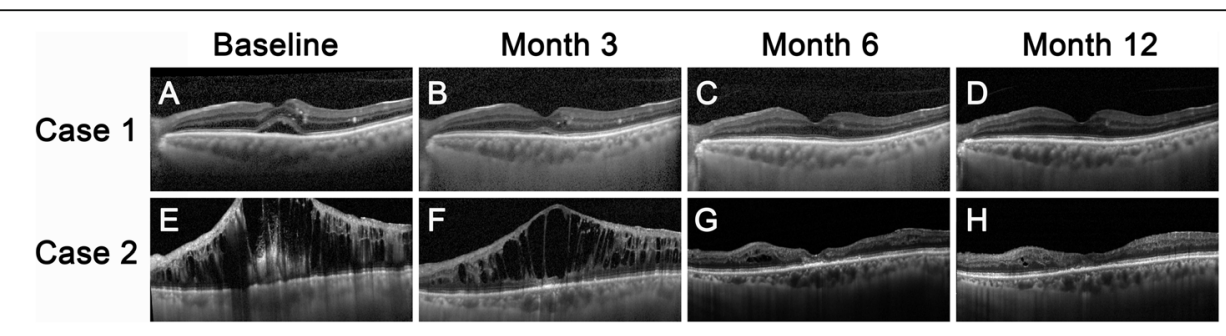

Fig. 3 Representative images of patients who received intravitreal injections of ranibizumab (IVR) under " $1+$ PRN" regimen. a-d were optical coherence tomography (OCT) images for case 1 at the time-points of baseline, month 3, month 6, and month 12, respectively. Case 1 had a bestcorrected visual acuity (BCVA) of 0.3 and intact of external limiting membrane (ELM) and ellipsoid zone (EZ) at baseline (a). The subretinal fluid was absorbed immediately after one injection of ranibizumab, and the BCVA increased to 0.6 at month 1 . No additional injection was needed for case 1, and the macular remained dry with the BCVA increased to 0.8 at month $12(\mathbf{b}-\mathbf{d})$. (E-H) were OCT images for case 2 at baseline, month 3 , month 6, and month 12, respectively. Please note that case 2 had a disrupted ELM and EZ with a BCVA of 0.1 at baseline (e). Intraretinal fluid was partially absorbed after three injections of ranibizumab (f). Case 2 continued to receive another three injections of ranibizumab monthly and then intraretinal fluid was totally absorbed at month 6 (g). After a total of 6 times IVR under " $1+$ PRN" regimen the intraretinal fluid was totally absorbed, however, the BCVA was still 0.1 at month 12 
Table 2 Multivariate analysis of predictive factors for final best corrected visual acuity

\begin{tabular}{|c|c|c|c|c|c|c|}
\hline \multirow[t]{2}{*}{ Variables } & \multicolumn{2}{|c|}{ Unstandardized coefficients } & \multirow{2}{*}{$\begin{array}{l}\text { Standardized coefficients } \\
\beta\end{array}$} & \multirow[t]{2}{*}{$P$} & \multicolumn{2}{|c|}{ 95\% confidence interval for B } \\
\hline & $B$ & SE & & & Lower bound & Upper bound \\
\hline Age & -0.005 & 0.002 & -0.233 & $0.013^{\mathrm{a}}$ & -0.009 & -0.001 \\
\hline Gender & 0.086 & 0.043 & 0.161 & 0.052 & 0.000 & 0.172 \\
\hline DM duration & 0.011 & 0.007 & 0.135 & 0.155 & -0.004 & 0.026 \\
\hline DME duration & -0.027 & 0.035 & -0.068 & 0.438 & -0.096 & 0.042 \\
\hline BCVA at baseline & 0.498 & 0.096 & 0.441 & $0.001^{\mathrm{a}}$ & 0.305 & 0.691 \\
\hline CFT & 0.000 & 0.000 & 0.000 & 0.994 & 0.000 & 0.000 \\
\hline Choroidal thickness & 0.000 & 0.000 & -0.046 & 0.586 & 0.000 & 0.000 \\
\hline Intraretinal cysts & 0.001 & 0.046 & 0.002 & 0.980 & -0.092 & 0.094 \\
\hline SRF & 0.034 & 0.047 & 0.066 & 0.471 & -0.060 & 0.129 \\
\hline PVD & -0.144 & 0.048 & -0.275 & $0.004^{\mathrm{a}}$ & -0.241 & -0.047 \\
\hline VMT & 0.231 & 0.078 & 0.284 & $0.005^{\mathrm{a}}$ & 0.075 & 0.388 \\
\hline ERM & 0.087 & 0.078 & 0.101 & 0.269 & -0.069 & 0.243 \\
\hline Disrupted EZ & 0.155 & 0.058 & 0.294 & $0.010^{\mathrm{a}}$ & -0.006 & 0.235 \\
\hline Disrupted ELM & 0.104 & 0.064 & 0.193 & 0.108 & -0.024 & 0.231 \\
\hline Disrupted RPE layer & -0.033 & 0.05 & -0.064 & 0.506 & -0.133 & 0.067 \\
\hline
\end{tabular}

SE standard error, DM diabetes mellitus, DME diabetic macular edema, BCVA best corrected visual acuity, CFT central foveal thickness, SRF subretinal fluid, PVD posterior vitreous detachment, VTM vitreomacular traction, ERM epiretinal membrane, EZ ellipsoid zone, ELM external limiting membrane, RPE retinal pigment epithelium

${ }^{a}$ Statistically significant result

(DRCR.net) study [7, 21, 22], the RESTORE study [9], the REVEAL study [23], RISE and RIDE study [24], and the REFINE study [11], have demonstrated the safety and effectiveness of IVR for treating DME. In some previous clinical trials, continuous monthly injections of ranibizumab has been recommended, which may optimize the efficacy of treatment [25]. However, monthly injections are not practical in the real world, therefore, ophthalmologists are now seeking other alternative treatment regimens. Three loading doses of antiVEGF injections followed by a PRN protocol is now widely adopted by clinicians for treating center-involved DME [11, 25, 26]. However, three loading injections could still be a great economic and psychological burden for patients with DME, especially for those who live in developing countries. Meanwhile, a proportion of patients actually do not need the three loading injections and yet maintain good vision over long period [27], therefore, for these patients three loading doses may be unnecessary and would be a waste of money. Recently, ophthalmologists are focus on other treatment modalities like the treat-and-extend regimen [28] and " $1+$ PRN" regimen for the management of DME.

There are still very few studies evaluating the effectiveness of " 1 + PRN" regimen for treating center-involved DME. In our study, we demonstrated that more than one third of the cases did not need three loading doses at month 3, which was consistent with the findings showed by James et al. [26]. James reported that 24 out of 180 eyes (13\%) need only one injection of ranibizumab, 52 out of 180 eyes underwent 2 injections, and the left 104 eyes had 3 injections on a monthly basis [26]. Therefore, James considered that there would be considerable reduction in health care cost if one third of patients did not received the three loading doses for treating DME [26]. Further, in a recent retrospective study, Ebneter et al. compared outcomes of PRN injections based on BCVA versus OCT-based treat-andextend regimen for DME, and they found that two groups had similar visual acuity outcomes, but patients in the PRN injections group based on BCVA received significantly fewer intravitreal injections than patients in the OCT-based treat-and-extend regimen group [28]. Specifically, the mean number of the IVR was 6.83 times during the one-year follow-up visit in our study, which was similar with the mean number of 5 times reported by James et al. under " $1+$ PRN" regimen [26]. Interestingly, this mean number of 6.83 injections under " $1+$ PRN" regimen in our study was relatively fewer than the number of 7.9 injections reported by REFINE study [11] and average 7 injections reported by RESTORE study under "3 + PRN" regimen [9]. In our study, the logMAR VA improved from 0.64 before treatment to 0.46 at the final visit of month 12, namely increasement of $0.18 \mathrm{log}$ MAR VA, which was similar with " $3+$ PRN" studies such as REFINE study [11] and RESTORE study [9] but lower than the increasement of 0.29 logMAR VA under monthly injection regimen reported by Nepomuceno et al. [29]. In 
addition, in our study, the mean CFT decreased from $478.23 \mu \mathrm{m}$ before treatment to $270.39 \mu \mathrm{m}$ at final visit of month 12, which was a reduction of $207.84 \mu \mathrm{m}$ of CFT for the patients over one-year " $1+$ PRN" treatment. Interestingly, the mean changes of CFT varied in different studies with different treatment regimens. The REFINE study showed a mean reduction of $146.5 \mu \mathrm{m}$ for CFT after 12 months' treatment under " $3+\mathrm{PRN}$ " regimen [11]. The RISE and RIDE study reported a mean reduction of $249.3 \mu \mathrm{m}$ for CFT after monthly intravitreal injections of ranibizumab for 12 months [30] while Nepomuceno et al. reported a mean reduction of $126 \mu \mathrm{m}$ for CFT under monthly injection regimen [29]. We believed that the mean changes of BCVA and CFT varied not only related to the treatment regimens but also related to other factors such as inclusion criteria and exclusion criteria. Therefore, future randomized controlled clinical trials are still needed to compared the efficacy between " $1+$ PRN" and other treatment regimens.

It is important for the ophthalmologists to know whether baseline characteristics would predict the final visual outcome for the patients treated by the ranibizumab. Sophie et al. found that good baseline BCVA was a predict factor for final BCVA of 20/40 or better, and submacular fluid and severe cystic edema were predict factors for poor visual outcome without treatment but respond well when treated with monthly IVR [31]. Channa et al. revealed that poor baseline BCVA predicted poor visual outcomes [32]. Yucel et al. reported that older age, female, poor BCVA at baseline, VMT, and disruption of EZ were predictors for final poor BCVA prognosis, while PVD and leaking microaneurysms were predictors for the good final BCVA [27]. Our "1 + PRN" regimen data had similar results with previous studies [27, 31-33]. Interestingly, in our study we found it that although there was statically difference between the SFCT at baseline and SFCT at 12 months' follow-up time-point after intravitreal injections of ranibizumab under " $1+$ PRN" regimen, multivariate linear regression analysis demonstrated that SFCT at baseline was not a predictive factor for BCVA at the final visit of month 12 . Actually, there is still controversy on the role of SFCT for predicting the VA outcome. Rayess et al. reported that greater baseline SFCT were associated with better anatomic and functional responses [34]. Similarly, Nourinia et al. reported in their prospective interventional case series that SFCT reduction was correlated with CFT reduction as well as vision improvement [35]. However, Campos et al. found that baseline SFCT decreased was not a predictor for anatomic or functional outcome for treatment of DME outcome [36]. In our study, although there was statically difference between the SFCT at baseline and SFCT at month 12 after intravitreal injections of ranibizumab under " $1+\mathrm{PRN}$ " regimen, multivariate linear regression analysis demonstrated that SFCT at baseline was not a predictive factor for BCVA at the final visit of month 12 . However, the role of SFCT still requires further investigations.

Our study has some limitations including relatively small number of patients and short-term follow-up visit. Besides, although we have full data of the patients treated with ranibizumab, there was no control group in our study. Even with these limitations, our results have enough strength to conclude that the intravitreal injections of ranibizumab under " $1+\mathrm{PRN}$ " regimen is a safety and effective way to improve BCVA and reduce the CFT of DME patients, which could be an option for the treatment of center-involved DME.

\section{Conclusion}

In summary, based on our results, intravitreal injections of ranibizumab under " $1+$ PRN" regimen could significantly improve BCVA and reduce the CFT and SCFT over 12 months. Older age, poor baseline BCVA, presence of VMT, and EZ disruption were more at risk of poor final VA, while development of PVD was associated with final good BCVA at one-year follow-up visit.

\section{Abbreviations \\ DME: Diabetic macular edema; VEGF: Vascular endothelial growth factor; PRN: Pro re nata; BCVA: Best-corrected visual acuity; CFT: Central foveal thickness; FFA: Fundus fluorescein angiography; OCT: Optical coherence tomography; EDI: Enhanced depth imaging; logMAR: Logarithm of minimal angle of resolution; RPE: Retinal pigment epithelium; SFCT: Subfoveal choroidal thickness; EZ: Ellipsoid zone; ELM: External limiting membrane; PDR: Proliferative diabetic retinopathy; SRF: Subretinal fluid; PVD: Posterior vitreous detachment; VMT: Vitreomacular traction.}

\section{Acknowledgements}

Not applicable.

\section{Authors' contributions}

$\mathrm{KL}$ and $\mathrm{CH}$ conducted the study, contributed to the data analysis, and drafted the paper. LL, YG, FX, XZ and LL made contributions to acquisition of data and drafting. CJ contributed to the design of the study, analysis for the data, and was responsible for revising the paper. All authors have read and approved the manuscript.

\section{Funding}

This study was supported by the Key Projects of Guangzhou Science and Technology Plan (201707020008), 5010 Project of Clinical Research of Sun Yat-sen University (2013007), the Science and Technology Planning Project of Guangdong Province (2017A020215038), National Natural Science Foundation of China (81600741), and Bethune Langmu Ophthalmological Research Fund for Middle-aged and Young People (BJ-LM2017004J). The funding sponsors were not involved in the design of the study and collection, analysis, and interpretation of data and in writing the manuscript.

\section{Availability of data and materials}

The datasets used and/or analysed during the current study are available from the corresponding author on reasonable request.

\section{Ethics approval and consent to participate}

This research conducted according to the tenets of the Declaration of Helsinki. Prior approval was obtained from the IRB of Zhongshan Ophthalmic Center of Sun Yat-sen University. Written informed consent was obtained from all enrolled subjects. 


\section{Consent for publication}

Not applicable.

\section{Competing interests}

The authors declare that they have no competing interests.

Received: 20 October 2019 Accepted: 9 June 2020

Published online: 15 June 2020

\section{References}

1. Resnikoff S, Pascolini D, Etya'ale D, Kocur I, Pararajasegaram R, Pokharel GP, et al. Global data on visual impairment in the year 2002. Bull World Health Organ. 2004;82(11):844-51.

2. Klein R, Klein BE, Moss SE, Cruickshanks KJ. The Wisconsin epidemiologic study of diabetic retinopathy: XVII. The 14-year incidence and progression of diabetic retinopathy and associated risk factors in type 1 diabetes. Ophthalmology. 1998;105(10):1801-15.

3. Man RE, Fenwick EK, Sabanayagam C, Li LU, Tey CS, Soon HJ, et al. Differential impact of unilateral and bilateral classifications of diabetic retinopathy and diabetic macular edema on vision-related quality of life. Invest Ophthalmol Vis Sci. 2016;57(11):4655-60.

4. Antonetti DA, Klein R, Gardner TW. Diabetic retinopathy. N Engl J Med. 2012;366(13):1227-39.

5. Zimmet PZ, Alberti KG. Epidemiology of diabetes-status of a pandemic and issues around metabolic surgery. Diabetes Care. 2016;39(6):878-83.

6. Early Treatment Diabetic Retinopathy Study research group. Photocoagulation for diabetic macular edema. Early treatment diabetic retinopathy study report number 1. Arch Ophthalmol. 1985;103(12):1796-806.

7. Elman MJ, Aiello LP, Beck RW, Bressler NM, Bressler SB, Edwards AR, et al. Randomized trial evaluating ranibizumab plus prompt or deferred laser or triamcinolone plus prompt laser for diabetic macular edema. Ophthalmology. 2010;117(6):1064-77 e1035.

8. Bressler NM, Varma R, Mitchell P, Suner IJ, Dolan C, Ward J, et al. Effect of Ranibizumab on the decision to drive and vision function relevant to driving in patients with diabetic macular edema: report from RESTORE, RIDE, and RISE trials. JAMA Ophthalmol. 2016;134(2):160-6.

9. Mitchell P, Bandello F, Schmidt-Erfurth U, Lang GE, Massin P, Schlingemann $\mathrm{RO}$, et al. The RESTORE study: ranibizumab monotherapy or combined with laser versus laser monotherapy for diabetic macular edema. Ophthalmology. 2011;118(4):615-25.

10. Boyer DS, Nguyen QD, Brown DM, Basu K, Ehrlich JS. Outcomes with asneeded Ranibizumab after initial monthly therapy: long-term outcomes of the phase III RIDE and RISE trials. Ophthalmology. 2015;122(12):2504-13 e2501.

11. Li X, Dai H, Han M, Li J, Suhner A, Lin R, et al. Efficacy and safety of ranibizumab $0.5 \mathrm{mg}$ in Chinese patients with visual impairment due to diabetic macular edema: results from the 12-month REFINE study. Graefes Arch Clin Exp Ophthalmol. 2019:257(3):529-41.

12. Kang HM, Koh HJ. Long-term visual outcome and prognostic factors after intravitreal ranibizumab injections for polypoidal choroidal vasculopathy. Am J Ophthalmol. 2013;156(4):652-60.

13. Azar G, Wolff B, Mauget-Faysse M, Rispoli M, Savastano MC, Lumbroso B. Pachychoroid neovasculopathy: aspect on optical coherence tomography angiography. Acta Ophthalmol. 2017;95(4):421-7.

14. Aiello LP, Avery RL, Arrigg PG, Keyt BA, Jampel HD, Shah ST, et al. Vascular endothelial growth factor in ocular fluid of patients with diabetic retinopathy and other retinal disorders. N Engl J Med. 1994;331(22):1480-7.

15. Antonetti DA, Barber AJ, Hollinger LA, Wolpert EB, Gardner TW. Vascular endothelial growth factor induces rapid phosphorylation of tight junction proteins occludin and zonula occluden 1. A potential mechanism for vascular permeability in diabetic retinopathy and tumors. J Biol Chem. 1999; 274(33):23463-7.

16. Ferrara N, Damico L, Shams N, Lowman H, Kim R. Development of ranibizumab, an anti-vascular endothelial growth factor antigen binding fragment, as therapy for neovascular age-related macular degeneration. Retina. 2006;26(8):859-70.

17. Yu L, Liang XH, Ferrara N. Comparing protein VEGF inhibitors: in vitro biological studies. Biochem Biophys Res Commun. 2011;408(2):276-81.

18. Nguyen QD, Shah SM, Heier JS, Do DV, Lim J, Boyer D, et al. Primary end point (six months) results of the Ranibizumab for edema of the mAcula in diabetes (READ-2) study. Ophthalmology. 2009;116(11):2175-81 e2171.
19. Nguyen QD, Shah SM, Khwaja AA, Channa R, Hatef E, Do DV, et al. Two-year outcomes of the ranibizumab for edema of the mAcula in diabetes (READ2) study. Ophthalmology. 2010;117(11):2146-51.

20. Massin P, Bandello F, Garweg JG, Hansen LL, Harding SP, Larsen M, et al. Safety and efficacy of ranibizumab in diabetic macular edema (RESOLVE study): a 12-month, randomized, controlled, double-masked, multicenter phase II study. Diabetes Care. 2010;33(11):2399-405.

21. Elman MJ, Bressler NM, Qin H, Beck RW, Ferris FL 3rd, Friedman SM, et al. Expanded 2-year follow-up of ranibizumab plus prompt or deferred laser or triamcinolone plus prompt laser for diabetic macular edema. Ophthalmology. 2011;118(4):609-14.

22. Elman MJ, Qin H, Aiello LP, Beck RW, Bressler NM, Ferris FL 3rd, et al, Intravitreal ranibizumab for diabetic macular edema with prompt versus deferred laser treatment: three-year randomized trial results. Ophthalmology. 2012;119(11):2312-8.

23. Ishibashi T, Li X, Koh A, Lai TY, Lee FL, Lee WK, et al. The REVEAL study: Ranibizumab Monotherapy or combined with laser versus laser Monotherapy in Asian patients with diabetic macular edema. Ophthalmology. 2015;122(7):1402-15.

24. Nguyen QD, Brown DM, Marcus DM, Boyer DS, Patel S, Feiner L, et al. Ranibizumab for diabetic macular edema: results from 2 phase III randomized trials: RISE and RIDE. Ophthalmology. 2012;119(4):789-801.

25. Demirel S, Argo C, Agarwal A, Parriott J, Sepah YJ, Do DV, et al. Updates on the clinical trials in diabetic macular edema. Middle East Afr J Ophthalmol. 2016;23(1):3-12.

26. James DGP, Mitkute D, Porter G, Vayalambrone D. Visual outcomes following Intravitreal Ranibizumab for diabetic macular edema in a pro re Nata protocol from baseline: a real-world experience. Asia Pac J Ophthalmol (Phila). 2019;8(3):200-5.

27. Eski Yucel O, Birinci H, Sullu Y. Outcome and predictors for 2-year visual acuity in eyes with diabetic macular edema treated with Ranibizumab. J Ocul Pharmacol Ther. 2019;35(4):229-34.

28. Ebneter A, Waldmeier D, Zysset-Burri DC, Wolf S, Zinkernagel MS. Comparison of two individualized treatment regimens with ranibizumab for diabetic macular edema. Graefes Arch Clin Exp Ophthalmol. 2017;255(3): $549-55$.

29. Nepomuceno AB, Takaki E. Paes de Almeida FP, Peroni R, Cardillo JA, Siqueira RC, et al. a prospective randomized trial of intravitreal bevacizumab versus ranibizumab for the management of diabetic macular edema. Am J Ophthalmol. 2013;156(3):502-10 e502.

30. Brown DM, Nguyen QD, Marcus DM, Boyer DS, Patel S, Feiner L, et al. Longterm outcomes of ranibizumab therapy for diabetic macular edema: the 36month results from two phase III trials: RISE and RIDE. Ophthalmology. 2013; 120(10):2013-22.

31. Sophie R, Lu N, Campochiaro PA. Predictors of functional and anatomic outcomes in patients with diabetic macular edema treated with Ranibizumab. Ophthalmology. 2015;122(7):1395-401.

32. Channa R, Sophie R, Khwaja AA, Do DV, Hafiz G, Nguyen QD, et al. Factors affecting visual outcomes in patients with diabetic macular edema treated with ranibizumab. Eye (Lond). 2014;28(3):269-78.

33. Hu Y, Wu Q, Liu B, Cao D, Dong X, Zhang L, et al. Comparison of clinical outcomes of different components of diabetic macular edema on optical coherence tomography. Graefes Arch Clin Exp Ophthalmol. 2019;257(12): 2613-21.

34. Rayess N, Rahimy E, Ying GS, Bagheri N, Ho AC, Regillo CD, et al. Baseline choroidal thickness as a predictor for response to anti-vascular endothelial growth factor therapy in diabetic macular edema. Am J Ophthalmol. 2015; 159(1):85-91 e81-83.

35. Nourinia R, Ahmadieh H, Nekoei E, Malekifar P, Tofighi Z. Changes in central choroidal thickness after treatment of diabetic macular edema with intravitreal bevacizumab correlation with central macular thickness and best-corrected visual acuity. Retina. 2018;38(5):970-5.

36. Campos A, Campos EJ. Do Carmo a, Patricio M, Castro de Sousa JP, Ambrosio AF, et al. Choroidal thickness changes stratified by outcome in real-world treatment of diabetic macular edema. Graefes Arch Clin Exp Ophthalmol. 2018;256(10):1857-65.

\section{Publisher's Note}

Springer Nature remains neutral with regard to jurisdictional claims in published maps and institutional affiliations. 